\title{
An Analytical Model of Animal Growth
}

\author{
V. L. Stass \\ doi: 10.19044/esj.2017.v13n27p1 URL:http://dx.doi.org/10.19044/esj.2017.v13n27p1
}

\begin{abstract}
The purpose of this study was to explain some aspects of ontogenetic growth in pigs by analysing the relationships between variables that are significant to the development of animals. The novelty of the study is a new modelling approach to the growth problem, with the attention that has been paid to both a new set of variables, and an analytical discrete-continuous hybrid model, innovative for the field. This is a first species-specific hybrid model for animal growth formulated in discrete-time difference equation technique. The efficiency of the model is not only due to the modelling technique but also due to a set of relevant variables, especially a feed conversion coefficient, which provides a link between macro and micro physiological scales. The model is based on functional relationships between relevant variables acquired from experimental data analyses, and field observations. The concept explains some aspects of growth in pigs from 30 $\mathrm{kg}$ to $600 \mathrm{~kg}$, which is considered the maximum individual weight for a boar, and further growth up to a species maximum weight. The model predicts that boar can reach their maximum individual weight of $600 \mathrm{~kg}$ when 6,40 years old and are required to consume $62,51 \mathrm{~kg}$ of feed to put on the last kilogram. The phenotypes that can attain their maximum individual weight go through bifurcation of the growth trajectory, a transform in the growth mode. After bifurcation, the smallest number of the phenotypes go on the growth trajectory that leads to a set of species maximum weights of over $1205 \mathrm{~kg}$, and the greatest number of phenotypes continue to live until aged 24,90 years, provided their maximum weights do not change. The study includes growth rate equations, identifies species maximum weight phenotypes, and produces insight into pig longevity. The results suggest that species maximum weight growth trajectories are phenotype-dependant. A modified discrete-time difference equation technique combined with standard continuum methods is an appropriate formalism to model ontogenetic growth in animals.
\end{abstract}

Keywords: Animal development; growth dynamics; maximum weight; life span; difference equation; hybrid model 


\section{Introduction}

\section{Ontogenetic growth}

In animal development, growth is an indispensable process, and is probably the most specific and variable trait studied that entails many if not all levels of individual phenome. Morphogenesis, reproduction, senescence, and many other biological events occur alongside or during the course of growth. Development is a continuous process, and sometimes the "trait" that we are interested in is actually the growth process itself (Rice, 2008). Numerous factors influence the growth of an individual. These are internal, such as inherited qualities and physiological particulars, and external, such as environmental conditions.

Over the last century, there has been moderate progress in the development of novel growth theories; however, a number of growth functions have been created (Dumas at al. 2008) and applied in industry (de Lange et al. 2001). At the same time, there are few if any models, designed to describe growth solely in pigs. In most cases, one of the considered theoretically universal animal growth functions has been used (Schinckel \& de Lange, 1996). The most frequently applied mathematical function to model growth in animals is reportedly the Gompertz equation (Strathe et al. 2008) and the von Bertalanffy equation is considered most suitable to model individual growth (Dumas et al. 2010). To evaluate pigs' genetic potential for growth in industrial conditions, the Gompertz equation was suggested (Wellock et al. 2004).

Although the present range of growth functions seems to serve the purpose well (Birkett \& de Lange, 2001), a call for new, advanced, biologically meaningful (Hirst \& Forster, 2013; Boukal et al. 2014), next generation models exists. A unified mechanistic theory of growth remains elusive, a synthetic explanation is still needed for how and why growth rates vary as body size changes (Sibly et al. 2015). The reason for this is the apparently insufficient levels of qualitative and quantitative understanding of the related growth processes the models reveal (Dumas et al. 2010). The purpose of modelling growth in animals is to integrate the existing knowledge about the process, and apply mathematical methods to identify the qualitative and quantitative features, which are as yet unknown, likely to remain out of sight, or prove empirically unapproachable.

\section{Animal growth models}

Despite a considerable amount of accumulated experimental data, understanding of the biological processes that cause and control growth is far from complete, and theoretical tools have not been developed to resolve the issues that need to be addressed (Houle et al. 2010). The tools are thought concepts, models or theories that can convert the accumulated experimental 
data into knowledge (Brenner, 2010). These concepts can be formulated either in verbal or in mathematical form. The mathematical formulation is preferable due to the clear advantage of yielding analytical analyses. Moreover, biological interactions and processes are often nonlinear, and this is where intuitive, verbal reasoning may let us down; whereas mathematical methods allow us to analyse diverse biological processes (Baker et al. 2009).

Many animal growth models currently used in science and industry are built upon an equation first invented by Pütter (Boukal et al. 2014). A range of such models entails the Pütter equation and several derivatives of it, such as the well-known growth functions the von Bertalanffy, the Gompertz, logistic, and some others. These growth functions were produced when solving ordinary differential equations, by which certain speculative proportionalities between growth rate and animal weight were specified, without reference to the biological species studied. The functions obtained after solving the equations were termed general or universal growth functions. The resulting models specify animal growth dynamics, based on what is considered reasonable or believed to be true.

While some authors have considered the von Bertalanffy's law as one of the most universal biological patterns (Sousa et al. 2008), others who are more sceptical have argued that the universal growth functions provide only retrospective curve fitting with little, if any biological insight (Pittroff and Cartwright, 2002). If the derived functions are sufficient and practicable for general purposes, they remain inadequate for scientific explication of the complex variables that cause the growth of numerous biological species. It follows that a different approach is required: at first focus on species-specific growth models and then, applying acquired knowledge, formulate a unified concept of growth across species. The study offers a first analytical model to implement this approach.

\section{Hybrid model for animal growth}

There are recognised difficulties in applying mathematical methods to model development in animals. Living organisms entail not only the notion of biological function or purpose but also the notion of growth and development that differentiates biology from other natural sciences. Moreover, growth and development facilitate the increase of structural and functional heterogeneity in animals. Growth is an essential process in development that has been identified as a significant factor in the production of spatial heterogeneity (Maini et al. 2012), which complicates its mathematical modelling.

The two most classical modelling techniques of biological phenomena are continuous-time, and discrete-time models. A number of biological objects do not fit either formalism; such systems contain 
heterogeneity, where some phenomena are continuous while some are discrete. The modelling of this kind of systems would then naturally involve two different parts, a continuous one in ordinary differential equations, and a discrete one in difference equations, yielding what is called a 'hybrid' system (Mailleret et al. 2009). Hybrid modelling in biology has mainly been applied to couple continuous and discrete formulations. Although hybrid models are not new, the methods and approaches keep on developing, as well as the field of applications that keep widening (Stéphanou \& Volpert, 2016). In this field, neither a hybrid model for animal growth was published nor an appropriate modelling technique specified. Hybrid dynamical systems combine evolution equations with state transitions; when the evolution equations are discrete-time, also called map-based, the result is a hybrid discrete-time system (Cao \& Ibarz, 2010).

This reasoning suggests a possible explanation for the potential efficiency of modelling in animal growth by the discrete time modelling technique. If animal growth phenotype is a rapidly changing and not smooth function with many variables, then evidently, a discrete time model can outline the coarse dynamics of the same process without taking into consideration some implicit microscopic level events that take place in the chosen discrete time unit.

Difficulties cause not only intrinsic heterogeneity of biological objects but also different growth rates of different organism parts. Accordingly, in order to go beyond the limitations the growth functions impose (Boukal et al. 2014), it is necessary to extend the present range of methods, and develop the existing potentials of hybrid models (Hasenauer et al. 2015). For this purpose, a modified discrete-time difference equation technique combined with standard continuum methods would be an appropriate formalism.

\section{The model's description}

In this study, a hybrid modelling technique is applied. This is an attempt to formulate and analyse a hybrid model of animal growth, which combines the well known, though modified discrete-time difference equation technique, and standard continuum methods.

The study provides a partial explication of the changes in traits associated with the growth under nonindustrial conditions. However, the variables considered in the study are chosen to represent the underlying biology, but direct measurements of these variables are often difficult in practice. The model is a species-specific exercise constructed by considering relationships between variables analysed in experiments and field observations, without the inclusion of any theoretical premise. 
In the model, discrete time and continuum modelling techniques are combined to set up a scheme, which develops as time proceeds, and therefore is considered as a dynamical system. A big advantage of the model is the absence of unknown speculative parameters; the parameter estimation problem does not exist. Most models for animal growth are based on events taking place at the macroscopic level. This model has variable $Z$, a feed conversion coefficient, which provides a link with microscopic levels metabolic processes and, as a result, a coupling of different biological scales has been established.

Mathematically, the model is formulated as a set of partial difference equations with discrete chronological time. A marked quality of the model is its formulation to enable the relevant information to be concentrated in a parameter, and not in the initial and boundary conditions, as is usually the case with models of continuum dynamics. Growth in the pig under nonindustrial conditions was modelled from $30 \mathrm{~kg}$ up to a species maximum weight.

\section{Material and methods}

\section{Data set}

The data set was obtained from experiments on growing domestic pigs, $\mathrm{LW}$, fed from $30 \pm 6 \mathrm{~kg}$ up to $96 \pm 4 \mathrm{~kg}$ live weight. The pigs were housed and fed under non-industrial conditions, either in a pig testing station or in research facilities. The animals were kept loose in groups of up to four to a pen, or individually in pens, fed a dry balanced feed with unlimited access to water contingent on the experiment design, ad libitum, or a constrained diet, in accordance with the current body weight, adjusting the feed quantity once a week. Besides the experimental data set, records available from research pig-breeding farms, as well as the wild pig data publicly available from some European national parks, were used.

The growth processes are discussed and modelled in terms of body weight, daily gain, and a feed conversion coefficient. The performance of a phenotype, a trait, is regarded as a biological function of the underlying causal factors. The identification of such factors or variables is a separate task to complete prior to formulating a model. The quality of the model is contingent upon the choice of the variables. The two main variables used in the model, parameter $K$, an invariant, and $Z$, a feed conversion coefficient are not conventional biological traits, cannot be directly measured in animals, and are dimensionless. 


\section{The model's variables}

' $M$ ' stands for an individual animal current live weight, measured in kilograms.

$M=\{M \in$ 品 $+\mid 30 \leq M \leq 600\}$, Individual maximum weight, $M=M x=600 \mathrm{~kg}$ ' $m$ ' is animal initial considered weight, measured in kilograms, $M \geq m, m_{0}=$ $30 \mathrm{~kg}$.

' $t$ ' is chronological current time, measured in days starting from animal birth. $t=\{t \in \mathbb{N} \mid 0<t<\infty\}, \Delta t=1,2,3 \ldots \mathrm{n}, \mathrm{n} \in \mathbb{N}$.

'to' is starting time, correspond $m_{\mathrm{o}}, t_{\mathrm{o}}=90$ days.

' $K$ ' is a parameter, nondimensional.

$K=\{K \in$ 噣 $+\mid 1 \leq K<11\}, K_{\mathrm{o}}=1$.

' $Z$ is a current feed conversion coefficient, dimensionless.

$Z=\left\{Z \in\right.$ 四 $\left.+\mid Z_{0} \leq Z<\infty\right\},(Z=\infty) \rightarrow\left(M=M_{\mathrm{x}}\right)$. $Z_{\mathrm{o}}$ correspond $m_{\mathrm{o}}$.

\section{Results}

\section{Growth up to $600 \mathrm{~kg}$}

Analysis of the experimental data identified some functional relationships between variables, which form the model's base. Parameter ' $K$ ' proved to be an invariant that is equal for the same-weight animals during rapid growth between $m_{\mathrm{o}}=30 \mathrm{~kg}$ and $M=100 \mathrm{~kg}$ regardless of individual pig daily gain. It is considered that the relationships hold up to $M_{\mathrm{x}}=600 \mathrm{~kg}$. The parameter ' $K$ ' has the following form:

$$
K=\frac{M \cdot t}{m_{o}\left(2 t-t_{o}\right)}
$$

Equation (1) may be written in many forms; however, the two following equations are usually used:

$$
\begin{aligned}
& \frac{M}{m_{o}}=2 K-1+\frac{\left(t-K t_{o}\right)}{t} . \\
& \frac{1}{m_{o}} \cdot \frac{\Delta M}{\Delta t}=\frac{\Delta K}{\Delta t}+\frac{K}{t} .
\end{aligned}
$$

From the same data set, the following relationship was found:

$$
\frac{t-K t_{o}}{t}=-\frac{(2 K-Z) \cdot(K-1)}{Z K} \text {. }
$$

Substituting (4) into (2) and after obvious transformations, one get

$$
\frac{1}{m_{o}} \cdot \frac{\Delta M}{\Delta K}=\frac{2 K+1}{K}-\frac{2}{Z} \text {. }
$$

From (1) and (5) follows: 


$$
\frac{\Delta K}{\Delta t}=\frac{1}{t} \cdot\left[\frac{Z K^{2}}{Z(K+1)-2 K}\right]
$$

In this section, equation (5) will be analysed. Equation (5) is valid not only for rapidly growing animals, but also for the animals that reached individual maximum weight, $M_{\mathrm{x}}$, but not species maximum weight. At this stage, it is necessary to calculate $Z_{x}$ to derive relevant relationships. As the first step, it is shown how to find parameter ' $K$ ' under the condition $M=M_{\mathrm{x}}=600 \mathrm{~kg}$. In formal notation: $\left.K\right|_{(M=M \mathrm{x})}=K_{\mathrm{x}}$. Data analyses acquired from pig-breeding farms where mature boars were kept proved that (7) converges as $M \rightarrow M x$, and the following limit holds:

$\lim _{M \rightarrow M x}\left(\frac{2 K-1}{K+1}\right)=\sqrt{3}$, where $\left.\left(K \rightarrow K_{\mathrm{x}}\right)\right|_{M \rightarrow M x}$.

It follows from (7) that under condition $\left(K=K_{\mathrm{x}}\right), K_{\mathrm{x}}=5+3 \sqrt{ } 3=10,19615$.

The next step is to calculate $Z \mathrm{x}, Z \mid(M=M \mathrm{x})(K=K \mathrm{x})=Z_{\mathrm{x}}$.

Substituting $K_{\mathrm{x}}$ and $M_{\mathrm{x}}$ into (5) one obtains $Z_{\mathrm{x}}=62,51 . Z_{\mathrm{x}}$ an analytical expression that will be presented in sections below. One should notice that $Z_{\mathrm{x}}$ and $K_{\mathrm{x}}$ are constants both linked to $M_{\mathrm{x}}=600 \mathrm{~kg}$. $Z_{\mathrm{x}}$ has a clear biological interpretation. It specifies the quantity of feed in kilograms needed for a boar to put on the last kilogram to reach its individual maximum weight $M_{\mathrm{x}}=600 \mathrm{~kg} . K_{\mathrm{x}}$ specifies the association between $M$ and $t$ at $M_{\mathrm{x}} . K_{\mathrm{x}}$ provides the means to calculate the time at which $M_{\mathrm{x}}$ was reached.

From $\left(K_{\mathrm{x}}=10,19615\right)\left(M_{\mathrm{x}}=600\right)$ follows that $t \mid(M=M \mathrm{x})(K=K \mathrm{x})=t_{\mathrm{x}}=6,40$ years, is in agreement with field data. This means that under the model conditions, boars can reach $M_{\mathrm{x}}$ in 6,40 years, consuming $62,51 \mathrm{~kg}$ of feed to put on the last kilogram. This result is experimentally testable.

It was shown that equation (5) applies not only to model weight dynamic during the period of rapid growth, but is also correct up to the point an animal reaches maximum weight, $M$ x.

After individual maximum weight, $M_{\mathrm{x}}$ is reached, animals do not grow. It means that live weight $M_{\mathrm{x}}$ does not change and, as a result, feed conversion, $Z$ becomes infinity, $\left(t>t_{\mathrm{x}}\right) \mid(M=M \mathrm{x}) \rightarrow(Z=\infty)$. Under the above constraint, and considering equation (5), it follows:

$\lim _{z \rightarrow \infty}\left(\frac{1}{m_{o}} \cdot \frac{\Delta M}{\Delta K}\right)=\frac{2 K+1}{K}$, where $\left(K \rightarrow K_{1}\right) \mid z \rightarrow \infty$

From (8) under condition $\left(K=K_{1}\right)$ follows, $K 1=\left.K\right|_{(M=M \mathrm{x})(Z=\infty)=}$ 10,04975 . This result allows calculation of the time that corresponds to $K_{1}$, presented below.

It follows from $\left(M=M_{\mathrm{x}}\right)\left(K=K_{1}\right)$, that $t \mid(M=M \mathrm{x})(K=K 1)=t 1=24,90$ years. The time $t 1$ may be named 'obtainable life span'. The main condition to reach $t_{1}$ is that $M_{\mathrm{x}}$ remains constant between $t_{\mathrm{x}}$ and $t$. It is possible to show that not 
all phenotypes can reach $t_{1}$. Those phenotypes that can achieve their individual maximum weight, $M_{\mathrm{x}}$ can live until aged 24,90 years, provided their live weight $M_{\mathrm{x}}$ remain constant. However, $t_{1}$ is not a species maximum life span. It follows from the model, that theoretical maximum life span in the pig is 49,31 years, not analysed in this article.

The following relationship was found from data set analyses:

$\frac{t}{t_{o}}=\frac{2 K-1}{K+1} \cdot \Delta Z+1$

Results found earlier suggest that if the following condition holds $\left.t\right|_{(M=M x)\left(K=K_{1}\right)}=t_{1}$, than follows

$\frac{t}{t_{o}}=\frac{2 K-1}{K+1} \cdot \Delta Z+1 \rightarrow K^{2}, \quad \lim _{K \rightarrow K 1}\left(\frac{t}{t_{o}}\right)=K_{1}^{2}$.

It follows from (9) that $\left.t\right|_{K \rightarrow K_{l}}=t_{1}, t_{1} / t_{\mathrm{o}}=K_{1}^{2}$.

Considering equations (1), (5) and (6), it follows

$\frac{1}{m_{o}} \cdot \frac{\Delta M}{\Delta t}=\frac{1}{t} \cdot \frac{m_{o}[Z(2 K+1)-2 K]}{m_{o}[Z(2 K+1)-2 K]-M Z}$.

The condition that (10) reaches maximum is: $m_{o}(Z(2 K+1)-2 K)-M Z=0$. It means that the relationship that maximises (10) is

$$
\frac{M}{m_{0}}=2 K+1-\frac{2 K}{Z}, Z_{o}=1 \text {. }
$$

On the other hand, taking into consideration (10) one can see that there is no growth if $\mathrm{Z}(2 \mathrm{~K}+1)-2 \mathrm{~K}=0$. It means the condition under which animals do not grow at $m_{o}$ is $Z_{o}=2 / 3$. This is an unexpected result, counterintuitive and allegedly puzzling, attributable to the nonlinearity of the growth process. Formal, linear, empirical logic says that $Z$ may not be less than 1. This fact has some important implications.

In the sections above it is demonstrated that in experiments, collected data analyses and the model provide correct relationships between variables. The identified relationships extended beyond the experimental data domain, show results consistent with field observations.

The model predicts that boar can reach an individual maximum weight, $M_{\mathrm{x}} 6,40$ years old. Phenotypes that can reach $M_{\mathrm{x}}$ can live up to $t_{1}=$ 24,90 years, provided $M_{\mathrm{x}}$ remains constant between $t_{\mathrm{x}}$ and $t_{1}$.

The constants: $K_{\mathrm{x}}, K_{1}, M_{x}, t_{\mathrm{x}}, t_{1}$, will be important tools in further analyses. Below, the main facts are summarised.

$$
\frac{M_{x}}{m_{o}}=2 K_{x}-\frac{4}{K_{x}}
$$




$$
\begin{aligned}
& \frac{M_{x}}{m_{o}}=2 K_{1}-\frac{1}{K_{1}} \\
& \frac{t_{x}}{t_{o}}=\frac{K_{x}^{2}}{4} \\
& \frac{t_{1}}{t_{o}}=K_{1}^{2} \\
& \frac{1}{m_{o}} \cdot \frac{\Delta M}{\Delta t}=\frac{1}{t} \cdot \frac{m_{o}[Z(2 K+1)-2 K]}{m_{o}[Z(2 K+1)-2 K]-M Z}, K \leq K \mathrm{x}, Z \leq Z \mathrm{x} . \\
& \frac{1}{m_{o}} \cdot \frac{\Delta M}{\Delta t}=\frac{1}{t} \cdot \frac{Z K(2 K+1)-2 K^{2}}{Z(K+1)-2 K}, \text { where } K \leq K \mathrm{x}, Z \leq Z \mathrm{x} . \\
& \frac{1}{m_{o}} \cdot \frac{\Delta M}{\Delta t}=\frac{1}{t} \cdot \frac{K_{x}\left(2 K_{x}+1\right)}{K_{x}+1}, \text { where } K=K \mathrm{x}, Z=\infty . \\
& \frac{1}{m_{o}} \cdot \frac{\Delta M_{x}}{\Delta t_{x}}=\frac{4}{t_{o}} \cdot\left[\frac{Z_{x}\left(2 K_{x}+1\right)-2 K_{x}}{K_{x} \cdot Z_{x}}\right] \cdot\left[\frac{K_{x}-1}{K_{x}^{2}-4}\right], \mathrm{Z}=\mathrm{Z}_{\mathrm{x}}, K_{x}>2 .
\end{aligned}
$$

Due to the definition of the feed conversion coefficient, between $Z_{\mathrm{x}}=$ 62,51 and $Z=\infty$ there is a tiny indefinable border. In simple words, after the instant $M \mathrm{x}$ is reached, $Z=\infty$. Passage to the limit procedure, for example $(Z \rightarrow \infty)$, should be regarded a mathematical abstraction, and not a workable definition.

\section{Growth up to species maximum weight}

In this section, species maximum weight will be defined. It is understandable that the species maximum weight is greater than $M$ x. However, I start with $M_{\mathrm{x}}$, considering it variable. Taking the $K_{\mathrm{x}}$ as variable into consideration as well, consider (1) and (14) as a system. The equation that follows is given by

$$
K_{x}^{2}-\frac{K_{x} \cdot M_{x}}{2 m_{o}}-2=0
$$

There is a standard procedure of how to find the maximum $M_{\mathrm{x}}$. The condition that $M_{\mathrm{x}}$ in (18) has maximum is $\mathrm{d} M_{\mathrm{x}} \backslash \mathrm{d} K_{\mathrm{x}}=0$. It follows $\max M_{\mathrm{x}}=$ $M_{\mathrm{xx} 1}=4 m_{\mathrm{o}} K \mathrm{x}=1223,538 \mathrm{~kg}$. A sufficient condition for the maximum to exist, is $\mathrm{d}^{2} M \backslash \mathrm{d} K^{2}<\left.0\right|_{(M=M \mathrm{x})(K=K \mathrm{x})}$, is fulfilled. There is an inflection point (IP) on the $M$ xx growth trajectory. From $\mathrm{d}^{2} M \mathrm{x} \backslash \mathrm{d} K_{\mathrm{x}}{ }^{2}=0$, it follows that inflection point, $M_{\mathrm{xx} \mid i p}=2 m_{\mathrm{o}} K_{\mathrm{x}}=611,769 \mathrm{~kg}$. The inflection point on the $M_{\mathrm{xx}}$ growth trajectory, most likely the last one, was expected. This time, $M_{\mathrm{xx} 1}=1223,538$ $\mathrm{kg}$ was found by applying continuous methods, and the same result will be obtained below by applying the difference equation technique. Similarly to 
in the analyses above, in searching for species maximum weight, $M_{\mathrm{x}}$ was considered variable, and accordingly $K_{\mathrm{x}}$ and $K_{1}$. Consider (12) and (13) a system; after multiplying and rearranging, it follows:

$\frac{1}{m_{o}} \cdot \frac{\Delta M_{x}}{\Delta K_{x}}=\frac{\left(M_{x} \cdot K_{x}+m_{o}\right)+m_{o}\left(4 K_{x}^{3}-10 K_{x}-5\right)}{m_{o}\left(4 K_{x}^{3}-5 K_{x}\right)-K_{x}\left(M_{x} \cdot K_{x}+m_{o}\right)}$.

The condition under which (19) reaches maximum is: $m_{o}\left(4 K^{3} x-5 K_{x}\right)-$ $K_{x}\left(M_{x} K_{x}+m_{o}\right)=0$. From this follows:

$\max M_{x}=M_{x x 3}=\frac{2 m_{o}\left(2 K_{x}^{2}-3\right)}{K_{x}}$

Numerically, $M_{\mathrm{xx} 3}=1205,884 \mathrm{~kg}$, is different to $M_{\mathrm{xx} 1}$. The average of $M \times x 1$ and $M_{\mathrm{xx} 3}$ is $M_{\mathrm{xx} 2}$, is given below:

$M_{x x 2}=\frac{m_{o}\left(4 K_{x}^{2}-3\right)}{K_{x}}$

Therefore, there are the following three species maximum weights, obtained by applying both continuous and discrete methods:

$M_{x x l}=4 m_{o} K_{x}=1223,538 \mathrm{~kg}$

$M_{x x 2}=\frac{m_{o}\left(4 K_{x}^{2}-3\right)}{K_{x}}=1214,711 \mathrm{~kg}$

$M_{x x 3}=\frac{2 m_{o}\left(2 K_{x}^{2}-3\right)}{K_{x}}=1205,884 \mathrm{~kg}$

I can remind the interested reader that the heaviest reported domestic boar was $1157 \mathrm{~kg}$ (Mayer, 2009).

It follows from the model that $K_{\mathrm{xx}}=10,09807$ is a new constant related to $M_{\mathrm{xx}}$. The relation between $K_{\mathrm{xx}}$ and $K_{\mathrm{x}}$ is given by

$K_{x x}=K_{x}-\frac{1}{K_{x}}$.

The related time, $t \mid(\mathrm{M}=\mathrm{Mxx})=t_{\mathrm{xx}}=12,69$ years. $t_{\mathrm{xx}} / t_{\mathrm{o}}=\left(K_{\mathrm{x}} \cdot K_{\mathrm{xx}}\right) / 2$.

A possible interpretation is that (21), (22) and (23) are species asymptotic maximum weight phenotypes. A most unusual feature was that in order to find out the phenotypes, a combination of discrete and continuous methods was needed. Neither of the methods can produce the result separately. It seems that the information about the phenotypes was spread over different biological levels, or different scales, and conceivably had diverse biological meanings. The next point to underline is the procedure by which the phenotypes were found. The essential starting point was in field observations found $M$ x. It was chosen as the most frequently observed boar maximum weight. However, species maximum weight, $M_{\mathrm{xx}}$ does not directly follow from $M$ x. Nevertheless, by considering $M_{\mathrm{x}}$ as variable, and applying 
both discrete and continuous methods, the phenotypes were identified. This implies that the biological information about species maximum weight is also present in those animal genomes, which never reach it. It is in line with general notions about the process, and suggests the existence of a gene or process that either stops or enables the trajectory $M_{\mathrm{x}} \rightarrow M_{\mathrm{xx}}$.

\section{Rapid growth}

The aim of this section is to concentrate on the rapid growth, which occurs between $30 \mathrm{~kg}$ and $100 \mathrm{~kg}$ live weight in domestic pigs. In this weight range, a maximum growth rate is observed, and during the rapid growth, physiological and anatomical traits undergo fast change that requires additional attention. General equations (10) and (16) for growth are inconvenient for this purpose; therefore, a restricted model for rapid growth was built.

The mathematical base for the rapid growth model is a system derived from (2) and (4). The following equation is a good approximation of rapid growth $M m_{o}=2 K-1$. However, to apply (2) and (4), the following system is to consider:

$$
\begin{aligned}
& \frac{M}{m_{o}}=2 K-1 \\
& \frac{t-K t_{o}}{t}=0 \\
& \frac{(2 K-Z)}{Z K}=0
\end{aligned}
$$

The system of (24), (25) and (26) equations suggests that equations (2) and (4) were rearranged and considered differently. From (24) follows

$$
\frac{\Delta M}{\Delta K}=\frac{2 M}{2 K-1}, 100>M \geq m_{\circ} \text {. }
$$

From (25) follows

$$
\frac{\Delta K}{\Delta t}=\frac{K^{2} \cdot t_{o}}{t^{2}}, 100>M \geq m_{o} \text {. }
$$

From (27) and (28) follows

$$
\begin{aligned}
& \frac{\Delta M}{\Delta t}=\frac{2 K^{2} m_{o} t_{o}}{t^{2}}, 100>M \geq m_{o} . \\
& \frac{\Delta M}{\Delta t}=\frac{2 M K^{2} t_{o}}{(2 K-1) \cdot t^{2}}, 100>M \geq m_{o} .
\end{aligned}
$$

Considering (25), (26) and (27) one more growth equation is given by

$$
\frac{\Delta M}{\Delta t}=\frac{2 K Z m_{o}}{(Z-2 K) t+Z K t_{o}}, Z>K, 100>M \geq m_{o} \text {. }
$$


The above growth rate equations (29), (30) and (31) are only some of many possible under the restricted model. Below, a more advanced approach will be shown, which entails speculation about the growth genetic aspect. Considering (27) and (30), it follows

$$
\frac{\Delta K}{\Delta t}=\frac{K^{2} t_{o}}{t^{2}}+\frac{t-K t_{o}}{g t t_{o}}, 100>M \geq m_{o} .
$$

In (32) ' $\mathrm{g}$ ' is a speculative parameter, regarded as a genetic factor, which is thought to specify a particular growth phenotype. In this paper, 'genetic factor' is not discussed further. From (27) and (32) follows

$$
\frac{\Delta M}{\Delta t}=\frac{2 m_{o}}{t}\left[\frac{K^{2} t_{o}}{t}+\frac{t-K t_{o}}{g t_{o}}\right], 100>M \geq m_{o},(g \in \mathbb{Z}) \vee(g \in \sqrt{\mathbb{Z}}+) \text {. }
$$

\section{Discussion}

\section{Growth dynamics}

It is widespread opinion that the growth rate in many mammals has a sigmoid form ( $\mathrm{Li} \& \mathrm{Wu}, 2010)$. This growth curve form is considered universal and is modelled by a number of growth functions (Barberis et al. 2011). The form results from growth dynamics, where it has one maximum and presumably two inflection points, either side of the maximum. In most cases, the rest of the curve is not specified, assuming it is smooth, monotonic, and asymptotically ends up at the animal maximum weight point.

This picture is tentatively accepted as a working hypothesis and has been frequently reproduced while modelling the growth of farm animals. The model can produce more precise growth dynamics, both in the rapid growth stage and in its final phase.

\section{Maximum weight}

The last part of the growth curve has a considerably different dynamic; the curve is neither smooth nor monotonic. There are two maximum animal weights, namely an individual one, and a species maximum one. They differ considerably, but only some phenotypes are biologically capable of reaching the species maximum weight.

Moreover, there is an inflection point on the growth trajectory between the two maximum weights. The inflection point suggests that the growth trajectory is modelled correctly, mathematically accurately, and biologically reasonable. At this inflection point, $\left(M_{\mathrm{xx}}\right.$ lip $)$ the growth cessation stops and the growth process starts again.

Under the model conditions, the phenotypes, which are able to reach their individual maximum weight, go through a transformation in the growth mode. It means that on the growth trajectory $M_{\mathrm{x}}$ at the point $\left(M_{\mathrm{x}}, Z_{\mathrm{x}}, K_{\mathrm{x}}, t_{\mathrm{x}}\right)$, 
bifurcation takes place with two new growth trajectories to develop. On one trajectory $\left(M \rightarrow M \times \rightarrow M \times\left.\right|_{1}\right)$, animals continue to live up to 24,90 years, provided their individual maximum weight, $M x$ remains unchanged. On another trajectory $\left(M \rightarrow M_{\mathrm{x}} \rightarrow M_{\mathrm{xx}}\right)$ phenotypes continue to grow to reach the species maximum weight, $M_{\mathrm{xx}}$. What happens when the animals reach individual maximum weight is that the growth temporarily stops until a new growth mode is initiated, which takes the animal development on the next possible trajectory from that starting point. An important precondition for the growth to continue $\left(M_{\mathrm{x}} \rightarrow M_{\mathrm{xx}}\right)$ is an inflection point on the growth trajectory. The inflection point can be thought of as a point on a curve, where the growth trajectory qualitatively changes. It takes approximately three months for the species maximum weight growth trajectory before it passes through the inflection point, $M_{\mathrm{xx}}$ ip. However, from the presented results it is difficult to deduce why growth stops, and starts after a while again, or how the growth mode has been changed; additional analyses is needed. The mechanism underlying growth dynamics regulation has been less well studied. There is little doubt that genes play an important part in the growth processes. However, little is known about how genes influence growth during development to shape animals of predetermined species-specific sizes.

\section{Growth variables}

Animal current weight, $M$ dynamics was modelled by parameter $K$, an invariant, and variable $Z$. Individual animal maximum weight, $M_{\mathrm{x}}$ is defined by parameter $K_{\mathrm{x}}$ and variable $Z_{\mathrm{x}}$; species maximum weight $M_{\mathrm{xx}}$ is modelled by the parameter $K_{\mathrm{x}}, K_{\mathrm{xx}}$ and variable $Z_{\mathrm{x}}, Z_{\mathrm{xx}}$ ensuring uniform modelling. variab

It suggests that parameter $K$ and le $Z$ are significant for modelling all stages of growth. Variable $Z$, a feed conversion coefficient, was not accounted for in theoretical studies. However, it not only provides a link with the microscopic levels of metabolic processes, but is also essential for modelling the dynamic of animal growth, and is indispensable to finding necessary facts. The model offers means for the direct calculation of $Z$. Although some calculations are possible, and some functional relations are clarified, the question about the animal weight balance equation is still open. This is partly due to the fact that the dynamic of variable $Z$ is sparsely understood. From the model it follows that the closer $Z$ is to unity, and the greater extent $(\Delta Z<0)$ the growth mode is developed to, the higher growth rate is. This seems reasonable; it is in line with feed conversion formal logic. However, equations (5) and (11) indicate the contrary. If in these equations $Z$ is set equal to 1 , a meaningless result is obtained. This suggests that the dynamic and functions of $Z$ are more complicated than was expected. 
Consider the result showing that there is no growth in the $Z_{o}$ range from $Z_{\mathrm{o}}=$ $2 \backslash 3, \ldots 3 \backslash 4, \ldots$ up to $Z_{o}=0,95$, that corresponds to the weight range from $m_{\mathrm{o}}$ to $M_{\mathrm{x}}$, respectively. If this logic is followed, there should be $\min Z, 0<Z<$ $2 / 3$, that would indicate the level of animal body conversion in order to maintain organism functions under animal weight decrease conditions. A growing organism is an autonomous open system, and can probably maintain its optimal functions in many alternative ways. Summarising the section above it is possible to state that the maximum growth rate is achievable not only when $Z$ tends to unity, but also under other, yet unknown conditions.

If $Z$ is a difficult variable, then parameter $K$ is more complicated. $K$ is remarkably informative and useful, however with complex dynamics. I shall briefly explain the general concept of applying parameter $K$. There are two options to utilise information in an open, autonomous system. The first option is dynamical, which entails definitions of initial and boundary conditions that entirely determine the process. The second option is parametrical, which entails nonspecific initial conditions, and not informative boundary conditions, and can be applied to a dissipative system with a stable trajectory. In this case, dynamic and structure of the system are completely determined by the parameter. However, the parameter or parameters should be informative enough to manage the process. The model is an attempt to implement this approach. Parameter $K$ and variable $Z$ are regarded as the parameters, which determine an animal's growth in the course of its life span. Parameter $K$ dynamic can be conditionally divided into two sets. One $K$ set $\left(K \rightarrow K_{\mathrm{x}} \rightarrow K_{\mathrm{xx}}\right)$ is associated with animal growth $\left(\mathrm{m} \rightarrow M \rightarrow M_{\mathrm{x}} \rightarrow M_{\mathrm{xx}}\right)$. These sets are tightly related to set $\left(Z \rightarrow Z_{\mathrm{x}} \rightarrow Z_{\mathrm{xx}}\right)$. The second $K$ set $\left(K \rightarrow K_{\mathrm{x}} \rightarrow K_{\mathrm{xx}} \rightarrow K_{1}\right)$ is associated with animal longevity $\left(t \rightarrow t_{\mathrm{x}} \rightarrow t_{\mathrm{xx}} \rightarrow t_{1}\right)$. This suggests that $K$ is sufficiently informative to model both longevity and growth. The above-mentioned sets are interrelated; however, the relation is not at all straightforward. Although there is insufficient understanding about dynamics of $K$ and $Z$, these variables are essential for modelling and analysing animal growth.

\section{Species maximum weight phenotypes}

Taking into consideration the above modelling results the following interpretation is proposed. If genetic determination of growth is denoted by the interaction of phenotypes $\mathrm{Y}^{\circ} \mathrm{P}^{\circ} \mathrm{G}$, the whole growth process, up to species maximum weight $M_{\mathrm{xx}}$ is obtained, in terms of phenotype sets is given by $\mathrm{Y}^{\circ} \mathrm{P}^{\circ} \mathrm{G} \Rightarrow \mathrm{W}, \mathrm{W}=\{\mathrm{AA}, \mathrm{Aa}$, aa $\} \mid \mathrm{M}=\mathrm{Mxx}$, where $\mathrm{W}$ is an ordered set of asymptotic species maximum weight phenotypes $\left\{M_{x x 1}, M_{x x 2}, M_{x x 3}\right\}$, respectively. This interpretation is in agreement with dissipative systems theory saying that dynamical system phase trajectories that start from diverse initial points end up in a single attractor. Under the model conditions, this 
attractor is the set $\mathrm{W}$. This suggests that in an animal phenome, the number of body weight phenotypes asymptotically reduces to one set with three species maximum weight phenotypes, regardless of the initial distribution of phenotypes. This result is consistent with the general notion about growth genetics and supports the opinion that genetic determination of phenotype changes during individual growth (Houle et al. 2010). It is in line with notions about the genetics of animal development, however the results above imply that the hypothesis according to which the combined action of many genes with small effects explains quantitative traits development is not the only one to consider. It seems that a limited number of genes can explain the development of a quantitative trait well.

The study fails to answer the question as to what determines species or an individual animal maximum weight. Species maximum weight was found considering the individual maximum weight as a variable. The causation is direct; it is the individual maximum weight that entails information about species maximum weight. This implies that the biological information about species maximum weight is also present in those animal genomes, which never reach it.

\section{Pig longevity}

Under the model conditions, pigs can reach the individual maximum weight, $M_{\mathrm{x}}=600 \mathrm{~kg}$ in 6,40 years; species maximum weight, $M_{\mathrm{xx}}$ in 12,69 years. Obtainable life span in the pig is 24,90 years, and species theoretical maximum longevity is 49,31 years. It suggests that most pigs can live barely longer than 25 years. This result is supported by field observations. There was only one reported case about a 27 year old pig (Hulbert et al. 2007). It follows from the model that the obtainable life span can be reached provided that the maximum individual weight $M_{\mathrm{x}}$ remains constant.

\section{Conclusion}

The study suggests that both rapid growth and species maximum weight growth trajectories are phenotype dependant. The biological information about species maximum weight is also present in those animal genomes, which never reach it.

The phenotypes, which are able to reach their individual maximum weight, go through bifurcation in the growth mode with two new growth trajectories to develop.

Species maximum weight is represented by a set with 3 asymptotic phenotypes, and is $1205,88 \mathrm{~kg}, 1214,71 \mathrm{~kg}$, or $1223,53 \mathrm{~kg}$.

Most pigs live less than 25 years, and only few phenotypes can live longer. 
A modified discrete-time difference equation technique combined with standard continuum methods is an appropriate formalism to model ontogenetic growth in animals.

The results can be considered as boundary conditions in the differential equations representing the lower, metabolic level processes.

The details of growth causation turn out to be so complex, and nonlinear that no animal weight balance equation can be formulated at this stage.

\section{References:}

1. Baker R.E, Schnell S. \& Maini P.K. (2009). Waves and patterning in developmental biology: vertebrate segmentation and feather bud formation as case studies. Int J Dev Biol. 53, 783-794.

2. Barberis L, Condat C.A. \& Román P. (2011). Vector growth universalities. Chaos, Solitons \& Fractals. 44, 1100-1105.

3. Birkett S. \& de Lange K. (2001). Limitations of conventional models and a conceptual framework for a nutrient flow representation of energy utilization by animals. British Journal of Nutrition. 86, 647659.

4. Boukal D.S, Dieckmann U, Enberg K, Heino M. \& Jørgensen C. (2014). Life-history implications of the allometric scaling of growth. Journal of Theoretical Biology. 359, 199-207.

5. Brenner S. (2010). Sequences and consequences. Philosophical Transactions of the RoyalSociety B. 365, 207-212.

6. Cao H. \& Ibarz B. (2010). Hybrid discrete-time neural networks. Philosophical Transactions of the Royal Society A. 368, 5071-5086.

7. Dumas A, Dijkstra J. \& France J. (2008). Mathematical modelling in animal nutrition: a centenary review. The Journal of Agricultural Science. 146, 123-142.

8. Dumas A, France J. \& Bureau D. (2010). Modelling growth and body composition in fish nutrition: where have we been and where are we going? Aquaculture Research. 41, 161-181.

9. de Lange C.F.M, Marty B.J, Birkett S, Morel P. \& Szkotnicki B. (2001). Application of pig growth models in commercial pork production. Canadian Journal of Animal Science. 81, 1-8.

10. Hasenauer J, Jagiella N, Hross S. \& Theis F.J. (2015). Data-driven modelling of biological multi-scale processes. Journal of Coupled Systems and Multiscale Dynamics. 3, 101-121.

11. Hirst A.G. \& Forster J. (2013). When growth models are not universal: evidence from marine invertebrates. Proceedings of the Royal Society B. 280, 1546. 
12. Houle D, Govindaraju D. \& Omholt S. (2010). Phenomics: the next challenge. Nature Reviews Genetics. 11, 855-866.

13. Hulbert A.J, Pamplona R, Buffenstein R, Buttemer W.A. (2007). Life and Death: MetabolicRate, Membrane Composition, and Life Span of Animals. Physiol Rev. 87, 1175-1213.

14. Li Y. \& Wu R. (2010). Functional mapping of growth and development. Biological Review. 85, 207-216.

15. Maini P.K, Woolley T.E, Baker R.E, Gaffney E.A. \& Lee S.S. (2012). Turing's model for biological pattern formation and the robustness problem. Interface Focus. 2, 487-496.

16. Mailleret L. \& Lemesle V. (2009). A note on semi-discrete modelling in the lifesciences. Philosophical Transactions of the Royal Society A. 367, 4779-4799.

17. Mayer J.J. (2009). Biology of wild pigs: wild pig physical characteristics. In Wild PigsBiology, Damage, Control Techniques and Management. (eds. J.J. Mayer and I.L Jr Brisbin), pp 25-51. Savannah River National Laboratory, US.

18. Pittroff W. \& Cartwright T.C. (2002). Modeling livestock systems. II. Understanding the relevant biology. Arch Latinoam Prod Anim. 10(3), 206-218.

19. Rice S.H. (2008). Theoretical Approaches to the Evolution of Development and Genetic Architecture. Ann N.Y. Acad Sci. 1133, 67-86.

20. Stéphanou A. \& Volpert V. (2016). Hybrid Modelling in Biology: a Classification Review. Math Model Nat Phenom. 11, 37-48.

21. Schinckel A.P. \& de Lange C.F. (1996). Characterization of growth parameters needed as inputs for pig growth models. Journal of Animal Science. 74, 2021- 2036.

22. Strathe A.B, Danfær A, Sørensen H. \& Kebreab E. (2010). A multilevel nonlinear mixed-effects approach to model growth in pigs. Journal of Animal Science. 88, 638-649.

23. Sibly R.M, Baker J, Grady J.M, Luna S.M, Kodric-Brown A, Venditti C. \& Brown J.H. (2015). Fundamental insights into ontogenetic growth from theory and fish. PNAS. 112, 13934-13939.

24. Sousa T, Domingos T. \& Kooijman S.A.L.M. (2008). From empirical patterns to theory: a formal metabolic theory of life. Philosophical Transactions of the Royal Society B. 363, 2453-2464.

25. Wellock I.J, Emmans G.C. \& Kyriazakis I. (2004). Describing and predicting potential growth in the pig. Animal Science. 78, 379-388. 